\title{
Clinical and audiometric features of presbycusis in Nigerians
}

\author{
*Sogebi OA1 ${ }^{1}$, Olusoga-Peters $\mathrm{OO}^{2}$, Oluwapelumi $\mathrm{O}^{2}$
}

1. Department of Surgery, College of Health Sciences, Olabisi Onabanjo University, Sagamu. Nigeria.

2. Department of Ear, Nose and Throat, Olabisi Onabanjo University Teaching Hospital, Sagamu, Nigeria

\begin{abstract}
Background: Presbycusis is the most common sensory impairment associated with ageing and it presents with variability of symptoms. Physicians need to recognize early clinical and audiometric signs of presbycusis in order to render adequate and quality care to patients and reduce associated morbidities.

Objective: To characterize the clinical modes of presentation and the typical audiometric tracings among patients with presbycusis.

Methods: This descriptive, prospective hospital-based study was conducted in the Ear, Nose and Throat (ENT) clinic of Olabisi Onabanjo University Teaching Hospital, (OOUTH) Sagamu, Nigeria. Patients with clinical diagnosis of presbycusis confirmed with bilateral sensorineural hearing loss (SNHL) on diagnostic audiometry were administered with questionnaires. Information obtained was analyzed using SPSS statistical package version 17.0 and presented in descriptive forms as percentages, means and graphs.

Results: Sixty-nine patients were diagnosed with presbycusis (M:F =1.6:1). Modal age group was 71-80 years. Hearing loss $88.4 \%$, tinnitus $79.7 \%$ and vertigo $33.3 \%$ were the major symptoms on presentation. The average duration of symptoms before presentation was 2.6 years. There was positive history of ototoxic drugs usage in $24.6 \%$, family history in $11.6 \%$, hypertension in $34.8 \%$ and osteoarthritis in $13.0 \%$. The most common type of audiometric pattern was strial. Hearing losses increased with age both at the speech and at the higher frequencies of sounds.

Conclusions: We found hearing impairment affected both speech and higher frequencies and the strial type of audiometric pattern was most common. The need for screening for hearing impairment from early middle age in symptomatic individuals is emphasized.

Keywords: Presbycusis, Nigerians, Audiometry, Clinical features, Elderly

African Health Sciences 2013; 13(4): 886 - 892 http://dx.doi.org/10.4314/ahs.v13i4.4
\end{abstract}

\section{Introduction}

Presbycusis is an age-related hearing loss mainly due to degenerative changes in the hair cells of the cochlea and central auditory connections ${ }^{1}$. It manifests with hearing impairment, poor speech discrimination and disruption of auditory processing in the central nervous system. Consequently most patients develop relative alienation from the environment and may be prone to emotional disturbances. Presbycusis is the most common sensory impairment associated with ageing and has prevalence of about $35 \%$ between ages 60 and 70 years ${ }^{2}$. The global increase in the average life expectancy including those of the developing countries suggests that the population of the elderly is on the increase with a projected increase in the incidence and prevalence of

*Corresponding author:
Dr. Olusola A Sogebi
ENT Unit, Department of Surgery
College of Health Sciences, Olabisi Onabanjo
University, Sagamu, Nigeria
Telephone: +234802326 2034
E-mail: ayosogebi2000@yahoo.com

presbycusis. Previously regarded as part of normal ageing process, there is scientific evidence that presbycusis may be another preventable age-related disorder ${ }^{3}$ if certain risk factors are eliminated or controlled.

Hearing loss of varying degrees remains the most common form of presentation; however there is wide variability of symptoms. It is important that physicians recognize early symptoms and signs of presbycusis in order to render adequate and quality care to the patients and reduce the morbidity associated with this disease.

Simple audiological evaluation like pure tone audiometry (PTA) and speech audiometry are amongst the tests for the diagnosis of presbycusis. The characteristic audiogram reveals bilateral symmetrical sensorineural hearing loss worse at the higher frequencies ${ }^{1}$. Left untreated, presbycusis can lead to isolation, dependence and frustration and generally reduced quality of life even among care givers ${ }^{4}$. Early detection and interventions including surgeries and fitting of assistive hearing devices can lead to improved quality of life.

African Health Sciences Vol 13 Issue 4 December 2013 
Presbycusis has been well characterized in many developed countries but there is paucity of studies in the developing world, creating a need for this study.

This study will contribute to knowledge about hearing loss and ageing, and will particularly be useful in the fields of otology, audiology, geriatric medicine and general medical practice.

\section{Methods}

The protocol for this study was approved by OOUTH health research and ethics committee.

This descriptive, prospective, hospital-based study was done among sixty nine patients that were diagnosed with presbycusis between January 2007 and December 2010.

\section{Sampling method}

Consecutive patients with clinical diagnosis of presbycusis confirmed with bilateral SNHL on diagnostic pure tone audiometry (PTA) were recruited for the study. PTA was performed in a sound-proof booth, using a calibrated GSI67 diagnostic audiometer.

Questionnaires were administered to the patients and the information obtained included the sociodemographic characteristics, symptoms experienced, history of exposure to potentially harmful noise, family history of hearing impairment, use of alcohol and smoking, use of ototoxic drugs, presence of other chronic diseases like diabetes, hypertension, and osteoarthritis, and information on, and use of rehabilitative measures such as hearing aids.

\section{Inclusion/Exclusion}

Consent were obtained from the patients after the nature, the general content, including the fact that a decline to participate will not affect treatment was explained to the patients. Every patient that was too ill to withstand performing an audiogram and those who had severe impairment with communication and found it difficult to understand the questions were excluded.

\section{Data presentation and analysis}

The data obtained based on the information was fed into the spreadsheet and was analysed using the SPSS version 17, and the results were presented in descriptive forms as tables and graphs.

\section{Results}

There were sixty-nine patients diagnosed with presbycusis comprising of 42 males and 27 females (M: F =1.6:1). The ages ranged from 45 to 93 years with the modal age group of 71-80 years. The other sociodemographic characteristics are shown in table 1.

\section{Table1: Socio-demographic characteristics of the patients}

\begin{tabular}{lll}
\hline & $\begin{array}{l}\text { Male } \\
\mathrm{n}=42 \\
(60.9 \%)\end{array}$ & $\begin{array}{c}\text { Female } \\
\mathrm{n}=27 \\
(39.1 \%)\end{array}$ \\
\hline $\begin{array}{lll}\text { Age group (Years) } \\
<50\end{array}$ & $3(7.1)$ & $0(0.0)$ \\
$51-60$ & $7(16.7)$ & $3(11.1)$ \\
$61-70$ & $10(23.8)$ & $9(33.3)$ \\
$71-80$ & $17(40.5)$ & $12(44.4)$ \\
$>81$ & $5(11.9)$ & $3(11.1)$ \\
Mean & 68.7 & 71.7 \\
Level of education & & \\
No formal education & $2(4.8)$ & $1(3.7)$ \\
Primary & $7(16.7)$ & $5(18.5)$ \\
Secondary & $26(61.9)$ & $13(48.2)$ \\
Tertiary & $7(16.7)$ & $8(29.6)$ \\
Marital status & & \\
Married & $21(50.0)$ & $10(37.0)$ \\
Divorced/Separated & $8(19.1)$ & $3(11.1)$ \\
Widow (er) & $13(30.9)$ & $14(51.9)$ \\
Occupation & & \\
Unskilled & $5(11.9)$ & $3(11.1)$ \\
Skilled/ Professional & $12(28.6)$ & $6(22.2)$ \\
Retired & $25(59.5)$ & $18(66.7)$ \\
\hline
\end{tabular}

Apart from the hearing loss experienced by $88.4 \%$ of the patients, the other main symptom was tinnitus $(79.7 \%)$ while the average duration of symptoms before the patients presented to the hospital was 2.6 years. There was a history of use of ototoxic drugs in $24.6 \%$ of the patients while $11.6 \%$ had history of family member or relative with hearing impairment. Twenty four patients (34.8\%) were hypertensive, while $9(13.0 \%)$ had osteoarthritis as depicted in table 2 . 
Table 2: Clinical characteristics of the patients

\begin{tabular}{lc}
\hline Associated symptoms & $\mathbf{n}(\mathbf{\%})$ \\
\hline Hearing loss & $61(88.4)$ \\
Tinnitus & $55(79.7)$ \\
Vertigo & $23(33.3)$ \\
Otalgia & $17(24.6)$ \\
Fullness in the ear & $18(26.1)$
\end{tabular}

Duration of symptoms before presentation (years)

\section{Duration (years)}

$\begin{array}{ll}0.1-1.0 & 30(43.5) \\ 1.1-2.0 & 11(15.9) \\ 2.1-3.0 & 10(14.5) \\ 3.1-4.0 & 4(5.8) \\ 4.1-5.0 & 1(1.4) \\ >5.1 & 13(18.8)\end{array}$

Mean $=2.6+/-1.9$ years; $\quad$ Median $=2.0$ years

Possible risk factors

Positive Family history 8 (11.6)

Exposure to noise 15 (21.7)

Previous use of ototoxic drug 17 (24.6)

Alcohol consumption $\quad 10(14.5)$

Smoking $\quad 5(7.3)$

Hypertension $24(34.8)$

Diabetes $6(8.7)$

Osteoarthritis $9(13.0)$

Peptic ulcer disease $1(1.5)$

Glaucoma $1(1.5)$

Asthma $1(1.5)$
Table 3 shows the grading of the hearing loss in the better ear, and revealed that almost three-quarter $(73.9 \%)$ of the patients had at least moderate hearing loss. Figure 1 is the graphical representation depicting increased hearing thresholds with increase in age. This trend was observed both at the speech and the higher frequencies. The most common type of audiometric pattern seen the patients was strial while five patients had unusual and another 5 had asymmetrical audiometric patterns as shown in figure 2 .

Table 3: Grading of Hearing loss in the Better ear

\begin{tabular}{ll}
\hline Grade & $\mathbf{n} \mathbf{( \% )}$ \\
\hline Normal & $5(7.2)$ \\
Mild & $13(18.8)$ \\
Moderate & $18(26.1)$ \\
Moderately severe & $16(23.2)$ \\
Severe & $7(10.1)$ \\
Profound & $10(14.5)$ \\
\hline
\end{tabular}

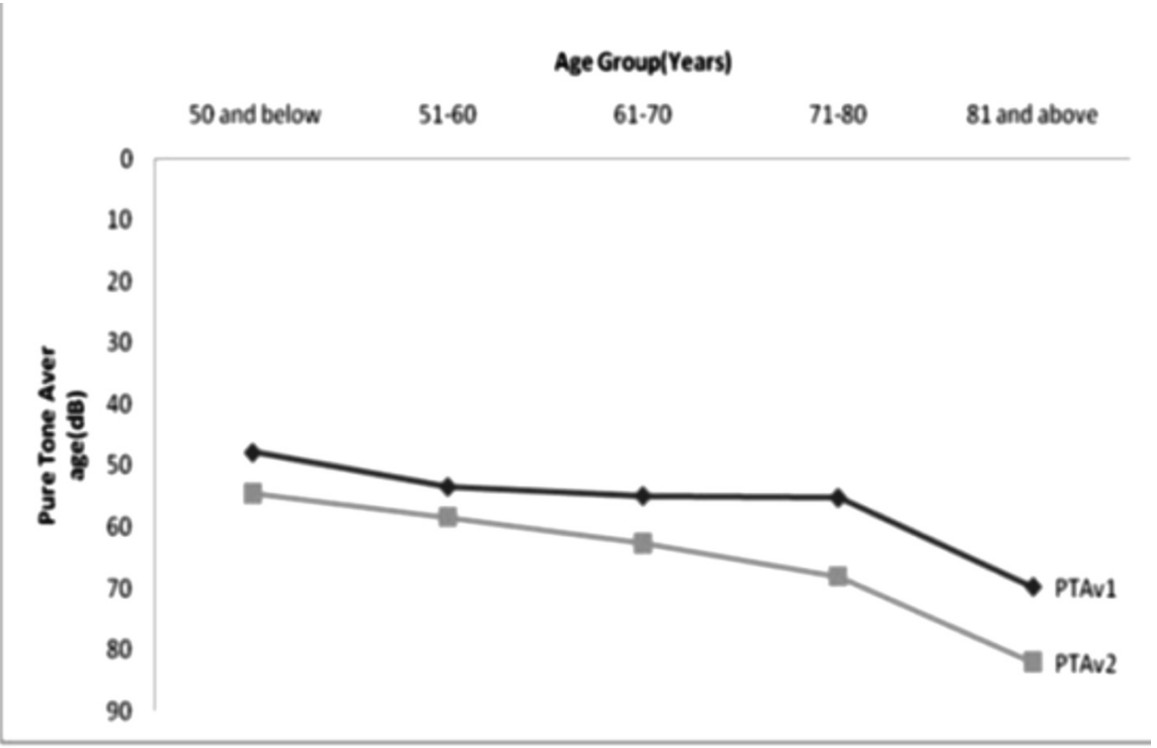

Figure1:Pure tone average Vs Age group 


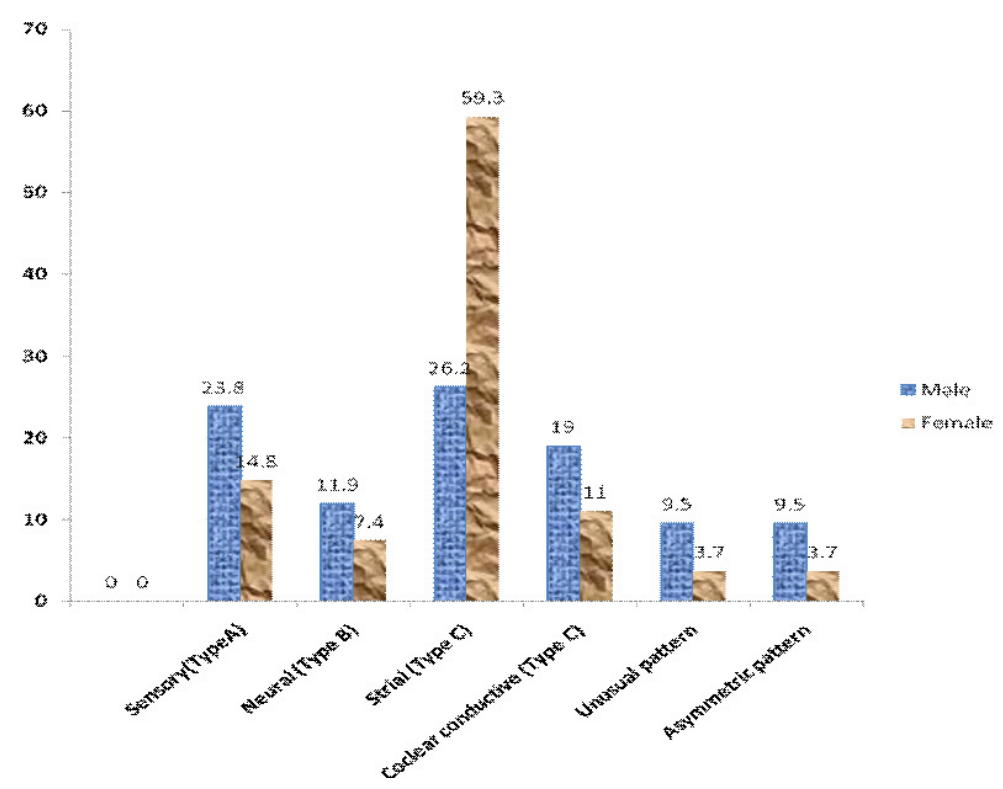

Figure 2: Types of audiograms

\section{Discussion}

From this study it appears that presbycusis tended to start manifesting about the fifth decade of life at the middle ages, progressed gradually over the years and peaked about the eighth decade, associated with distressing symptoms of tinnitus and vertigo, with most patients presenting within three years of noticing hearing impairment. Both speech frequencies and the high tones were affected.

Presbycusis has been described as a disease of the elderly due to degenerative changes in the cochlear hair cells and the neurons in the cochlear nerve and its central connections associated with ageing. Incidentally three of the patients (all males) in this study were within the fifth decade of life, the youngest being forty-five years. Ogunleye and Labaran in a previous study on patients in Ibadan had the youngest patient of forty-six years ${ }^{5}$. In the Great Britain MRC study on hearing loss, median hearing threshold levels in adults suggested that age related high frequency hearing decline commences after thirty years in men, but does not become apparent in women until after fifty years of age ${ }^{6}$. These are pointers that presbycusis may actually start at

earlier ages and progresses over the years. This assertion should however be made cautiously especially in the younger patients because presbycusis is a diagnosis of exclusion.

The hearing acuities also reduced with increasing age until after the eighth decade when there was a fall possibly due to fewer numbers of patients above this age. Similar findings were found in other studies ${ }^{7,8}$. Although there is a constant decline in hearing acuity with aging, age of onset, progression and severity of ARHI show great variation ${ }^{2}$. This connotes that with the increasing population of the elderly in the population, the burden of presbycusis will possibly increase. It is therefore reasonable to begin screening for presbycusis at younger ages in young adulthood ${ }^{9}$ particularly among symptomatic individuals.

It is also being suggested that presbycusis may be a preventable age-related disease like osteoarthritis, and cardiovascular diseases. Development of age-related hearing loss (ARHL) is determined by cumulative effects of individual's genetic predisposition and deleterious auditory exposures. While it may be difficult to modify genetic predisposition, it may be theoretically possible to prevent ARHL by minimizing ototraumatic exposures during the lifespan ${ }^{10}$. Environmental risk factors like exposure to noise, use of ototoxic drugs, head injuries are all modifiable. The use of alcohol and smoking were noted by some authors to be risk factors ${ }^{11,12}$ although these were not consistent. In the ISO 1999 study ${ }^{8}$ in which screened patients for the risk factors (noise exposure, ototoxic drug exposure, and otologic disease history) for ARHL were compared with the unscreened population, the unscreened population developed ARHL earlier and to a greater extent than the screened population, but both screened and unscreened subjects developed a 
hearing loss that began at the highest frequency measured $(6 \mathrm{kHz})$ and, with increasing age, expanded both in the magnitude and extent. Although this communication did not analyze the risk factors directly linked with presbycusis, it is worthy to note that some of the patients had positive family history, and history of adequate contact with some of the risk factors especially use of ototoxic drugs. Some of these drugs are across the counter analgesics such as

paracetamol, and non-steroidal anti-inflammatory drugs (NSAIDs) like acetylsalicylate which had been noted to increase the risk of hearing loss in men especially those younger than sixty years of age ${ }^{13}$.

Studies had also noted that the presence of other chronic medical conditions tended to worsen presbycusis $^{14}$. Many senior citizens are prone to chronic medical conditions some of which have simultaneous effect on their hearing. For example, chronic arteriosclerosis seen in patients with hypertension affect the arterial blood supply to the inner ear and consequently leads to hearing impairment. In patients with diabetes, two possible mechanisms had been muted; microangiopathic lesions in the inner ear could lead to cochlear hearing disorder or primary neuropathy of the cochlear nerve leading to retrocochlear hearing loss ${ }^{15}$. Hearing impairment could also be a consequence of the prolonged use of medication (some of which are ototoxic) in patients with chronic medical conditions ${ }^{16}$. The most common chronic medical condition reported by $34.8 \%$ of our patients was hypertension, followed by osteoarthritis $(13.0 \%)$.

Obviously less than half of our patients presented to the hospital within a year of noticing symptoms referable to the ear, with about one fifth of them presented over five years afterwards. Despite the relatively high level of education and enlightenment among the patients as above threequarter of the patients had at least secondary school education some patients still presented late (above one year). The level of education however will not automatically translate to early presentation as other factors such as presence of distressing symptoms and communication difficulties and functional impairment might have influence on the time of presentation. The symptoms of hearing impairment are usually insidious in onset, progresses gradually, and some individuals may not have difficulties in communication at speech frequencies except in particular circumstances such as in the noisy environment and during telephone conversation. This culminates into a tendency to watch if the hearing would improve with consequent delay in presentation. In a study in British Columbia, Canada, participants engaged in protracted self-assessment of their hearing, before, during and after seeking help for hearing problem ${ }^{17}$.

The main symptoms experienced by the patients were hearing loss (88.4\%), tinnitus $(79.7 \%)$, vertigo $(33.3 \%)$ and otalgia $(24.6 \%)$. This is similar to those found in other studies on the elderly with hearing impairment ${ }^{18-20}$. Each of these symptoms could make sufferers experience not only physical discomfort but could also affect them emotionally thus making life miserable. More distressing is the communication problems experienced by the patients. This is pertinent as hearing impairment affected the speech frequencies, although worse at the high tones. Elderly patients often have difficulties in hearing the high frequency sounds which may not be noticeable by people around, until they have difficulty in communication which is a sequel of affectation of the speech frequencies. Some authors have even observed involvement of the lower frequencies in certain pathologies. For instance, in a study in USA among patients with type 2 diabetes, Fristina et al found the greatest deficits at the lower frequencies ${ }^{21}$. It is therefore reasonable to suggest that although presbycusis affects the high frequency sounds at the outset, the hearing loss extends to the lower frequencies with time resulting in poor speech detection as well as poor speech understanding ${ }^{22}$. On the contrary some studies had found that the lower (including the speech frequencies) were relatively preserved in age-related hearing loss ${ }^{23,24}$ .These may be a result of variations in the study populations as many of such studies focused mainly on elderly individuals with hearing impairment and not necessarily presbycusis. Differences in the genetic constitutions of the various populations could also play a part in the factors.

The audiometry patterns was traditionally divided into four types (A-D) by Schuknecht in 1964 ${ }^{25}$, and further corroborated this in $1993{ }^{26}$ with pathologies in the temporal bones in a bid to describe and determine the pathology in the ear as follow:

(A) Sensory; a high-frequency hearing loss caused primarily by missing outer hair cells (OHCs);

(B) Neural; broad hearing loss with high-frequency emphasis and degeneration of the VIIIth nerve

(C) Strial; flat hearing loss with degeneration in stria vascularis (StV) and (D) Cochlear conductive; an 
evenly-sloping hearing loss hypothetically associated with changes in the stiffness properties of the basilar membrane.

Type $\mathrm{C}$ audiogram was the most common type found in this study similar to that in Ibadan, Nigeria ${ }^{5}$. This suggested that most pathology in our environment were due to the degeneration of the stria vascularis of the cochlea. At variance with the Ibadan study however, the other types of audiogram were sensory, cochlear conductive and neural in decreasing order. Five patients had unusual pattern i.e. bilateral symmetrical audiograms which did not fall into any of the four patterns described by Schuknecht, and another five patients with asymmetrical patterns i.e. disparity between the type of audiograms in the two ears. Katsarkas and Ayukawa noted only $50 \%$ of the audiograms in their study fitted into the described histopathological types, suggesting that in $50 \%$ of the subjects, presbycusis was caused by more than one degenerative process ${ }^{27}$. These imply that sometimes the cause of presbycusis may be multifactorial, and it may be difficult to attribute the cause to a particular factor because it is often a combination of deteriorated function of the auditory periphery and the central auditory system ${ }^{16}$. Asymmetric audiometric pattern may suggest a retrocochlear lesion like vestibular schwannoma. Further research concerning the risk factors for presbycusis is desirable in our environment.

Hearing aids is the most common form of rehabilitation for the hearing-impaired. Observations from the pure tone averages revealed that almost three-quarter of our patients had moderate hearing loss in the better ear and are candidates for hearing aids use. Only seventeen patients admitted that they had been counseled on the need for hearing aids, and nine of them actually procured hearing aids. However only three patients $5.9 \%$ of those needing aids) used their hearing aids regularly. In a study in Spain to assess the functional status of the elderly with hearing loss, only $4.5 \%$ of the participants who needed hearing aids used them regularly ${ }^{28}$. The relatively high cost of hearing aids and the exclusion of such facilities from the national health insurance scheme in Nigeria made the services not to be affordable to most patients who will need them.

Although a deficit in hearing can be managed to some degree by a hearing aid or other appropriate amplification devices, hearing-related rehabilitative needs are more than simply amplifying external sound ${ }^{29}$. Most of these patients are impoverished African Health Sciences Vol 13 Issue 4 December 2013 as they are dependent on their children and relatives for sustenance. There is an urgent need for the care of the elderly citizens for them to live more meaningful lives. In addition, family support and understanding will reduce the distress associated with hearing impairment in such patients.

\section{Conclusion}

The lack of comparative analysis and the risk factors for presbycusis were the major limitations of this study. However this study has increased the basic knowledge concerning this important subject in our environment and laid a template on which further researches can be built.

\section{References}

1. Rosenhall U. Presbyacusis-hearing loss in old age. Lakartidningen. 2001;98(23):2802-2806.

2. Fransen E, Lemkens N, Laer LV, Camp GV. Age-related hearing impairment (ARHI): environmental risk factors and genetic prospects. Experimental Gerontology. 2003; 38: 353-359.

3. Cruickshanks KJ, Nondahl DM, Tweed TS et al. Education, occupation, noise exposure history and the 10-yr cumulative incidence of hearing impairment in older adults. Hear Res. 2010;264(1-2):3-9.

4. Huang Q, Tang J. Age-related hearing loss or presbycusis. Eur Arch Otorbinolaryngol. 2010; 267(8):1179-1191.

5. Ogunleye AO, Labaran AO. Presbycusis in Nigerians at the University College Hospital, Ibadan. Afr J Med Med Sci. 2005;34(3):293-296.

6. Davis A. Hearing in Adults. Whurr Publishers Ltd. London. 1995. First ed London.

7. Baraldi GS , Almeida LC, Borges AC. Hearing loss in aging. Rev Bras Otorrinolaringol 2007; 73(1):64-70.

8. ISO 1999, 1990. Acoustics-Determination of occupational noise exposure and estimation of noise-induced hearing impairment. International Organization for Standardization.

9. Agrawal Y, Platz EA, Niparko JK. Prevalence of hearing loss and differences by demographic characteristics among US adults: data from the National Health and Nutrition Examination Survey, 1999-2004. Arch Intern Med. 2008; 168(14):1522-1530.

10. Bielefeld EC, Tanaka C, Chen GD, Henderson D. Age-related hearing loss: Is it a preventable condition? Hear. Res. 2010; 264(1-2): 98-107. 
11. Gopinath B, Flood VM, McMahon CM, Burlutsky G, Smith W, Mitchell P. The effects of smoking and alcohol consumption on agerelated hearing loss: the Blue Mountains Hearing Study. Ear Hear. 2010; 31(2):277-282

12. Itoh $\mathrm{A}, \mathrm{Nakashima} \mathrm{T}$, Arao $\mathrm{H}$ et al. Smoking and drinking habits as risk factors for hearing loss in the elderly: epidemiological study of subjects undergoing routine health checks in Aichi, Japan. Public Health. 2005; 115:192-196.

13. Curhan SG, Eavey R, Shargorodsky J, Curhan GC. Analgesic Use and the Risk of Hearing Loss in Men. The American Journal of Medicine 2010; 123 (3):231-237.

14. Agrawal Y, Platz EA, Niparko JK. Risk factors for hearing loss in US adults: data from the National Health and Nutrition Examination Survey, 1999 to 2002. Otol Neurotol. 2009;30(2):139-145.

15. Kurien M, Thomas K, Bhanu T S. Hearing thresholds in patients with Diabetes Mellitus. JLaryngol. Otol.1989; 103:164-168.

16. Mazelova J, Popelar J, Syka J. Auditory function in Presbycusis: peripheral vs. central changes. Experimental Gerontology. 2003; 38:87-94.

17. Carson A . What brings you here today? The role of self-assessment in help-seeking for agerelated hearing loss. Journal of Aging Studies 2005; 19: 185-200.

18. Ferreira LM, Ramos-Junior AN, Mendes EP. Characterization of tinnitus in the elderly and its possible related disorders. Braz. J Otorbinolaryngol. 2009; 75(2): 249-255.

19. Carmo LC, Silveira JA, Marone SA, D'Ottaviano FG, Zagati L, Lins EM. Audiological study of an elderly Brazilian population. Rev Bras Otorrinolaringol 2008;74(3):342-349.

20. Sogebi OA. Characterization of tinnitus in Nigerians. Auris Nasus Larynx. 2013; 40: 356360.

21. Frisina ST, Mapes F, Kim H, Frisina DR, Frisina RD. Characterization of hearing loss in aged type II diabetics. Hearing Research 2006; 211: 103-113.

22. Gates GA, Mills JH. Presbycusis. Lancet 2005; 366: 1111-1120.

23. Stenklev NC, Laukli E. Presbyacusis-hearing thresholds and the ISO 7029. Int J Audiol. 2004; 43:295-306.

24. Wiley TL, Chappell R, Carmichael L, et al. Changes in hearing thresholds over 10 years in older adults. J Am Acad Audiol. 2008; 19(4):281292.

25. Schuknecht HF. Further observations on the pathology of presbycusis. Arch. Otolaryngol. 1964; 80: 369-382.

26. Schuknecht, HF, Gacek MR. Cochlear pathology in presbycusis. Ann. Otol. Rhinol. Laryngol.1993; 102: 1-16.

27. Katsarkas A, Ayukawa H. Hearing loss due to aging (presbycusis). J Otolaryngol. 1986; 15(4):239-244

28. Hidalgo JL, Gras CB , Lapeira JT, Verdejo ML, Campo del Campo JL, Rabadan FE. Functional status of elderly people with hearing loss. Archives of Gerontology and Geriatrics 2009; 49: 88-92.

29. Liu XZ, Yan D. Ageing and hearing loss. J Pathol. 2007; 211(2):188-197. 